\title{
Decrease in Aryl Hydrocarbon Receptor and 17 $\beta$-Estradiol Receptor (A\&B) Gene Expression in The Hypothalamus and The Pineal Gland, After Administration of Dimethylbenz (A) Anthracene, A Mammary Carcinogen, To Sprague-Dawley Female Rats.
}

Thomas Cadoudal ${ }^{1,4}$, Véronique Lenoir ${ }^{2,4}$, Graziella Penot ${ }^{1,4}$, Indika SH Sathish ${ }^{3}$, Yueqin Zhao ${ }^{3}$, Xavier Coumoul ${ }^{1,4}$, Claude Forest $^{1,4}$ and Bernard Kerdelhue K, $^{*}$

${ }^{1}$ INSERM UMR-S 747, Université Paris Descartes, Pharmacologie Toxicologie et Signalisation Cellulaire, UFR Biomédicale, 45 Rue des Saints Pères, 75006 Paris, France

${ }^{2}$ Chemistry and Biology, Nucleo(s)tides and Immunology for Therapy (CBNIT), CNRS UMR 8601, Université Paris Descartes, PRES Sorbonne Paris Cité, UFR Biomédicale, 45 Rue des Saints-Pères, 75270 Paris Cedex 06, France

${ }^{3}$ Epidemiology and Biostatistics CORE, Eastern Virginia Medical School, Norfolk, USA

${ }^{4}$ Université Paris Descartes, Sorbonne Paris Cité, Paris, France

${ }^{*}$ Corresponding author: Bernard Kerdelhue, Université Paris Descartes, Neuroendocrinologie, Chemistry and Biology, Nucleo(s)tides and Immunology for Therapy (CBNIT), CNRS UMR 8601, Université Paris Descartes, PRES Sorbonne Paris Cité, UFR Biomédicale, 45 Rue des Saints-Pères, 75270 Paris Cedex 06, France, Tel: + 331 42864094; E- mail: bernard.kerdelhue@parisdescartes.fr

Rec date: Feb 27, 2014, Acc date: Mar 31, 2014, Pub date: Apr 05, 2014

Copyright: @ 2014 Cadoudal T, et al. This is an open-access article distributed under the terms of the Creative Commons Attribution License, which permits unrestricted use, distribution, and reproduction in any medium, provided the original author and source are credited.

\begin{abstract}
Purpose: The promotion of mammary adenocarcinoma induced by Dimethyl benz (a) Anthracene (DMBA) is preceded by disruptions of the Hypothalamo-Pituitary-Gonadal and Hypothalamo-Pituitary-Adrenal axes and by a reduction of the secretion of Melatonin. We hypothesized that these disruptions might be induced by changes, in neuroendocrine structures of the brain, in the gene expression of receptors for Aryl Hydrocarbon (AHR) and17ßEstradiol (ERS1 and ERS2).

Methods: Sprague Dawley female rats received one single administration of DMBA $(75 \mathrm{mg} / \mathrm{kg})$ at $52-55$ days of age and were ovariectomized 5 days later. Then, one month later, RNAs from the Pineal Gland, the Hippocampus and the Hypothalamus were prepared and analyzed by real-time PCR for the presence of the transcripts encoding receptors for AHR and $17 \beta$-Estradiol (ERS1 and ERS2) together with those encoding Gonadotropin Releasing Hormone (GNRH1), Corticotropin Releasing Hormone (CRH) and Hydroxy-Indol-O-Methyl-Transferase (ASMT), the rate limiting enzyme for the synthesis of Melatonin.
\end{abstract}

Results: There was a long lasting and almost identical decrease in the expression of AHR, ERS1 and ERS2 in the Hypothalamus and the Pineal Gland. Also, there was an increase in the expression of ASMT and GNRH1 genes in the Pineal Gland, and of the GNRH1 gene in the Hypothalamus. However and, very interestingly, no effect was seen for the expression of any investigated gene in the Hippocampus, a structure of the brain implicated in cognition.

Conclusion: DMBA reduces the brain expression of AHR, ERS1 and ERS2, key transcriptional factors, but only in brain structures involved in the control of neuroendocrine systems.

Keywords: AHR; ERS1; ERS2; Gene expression; DMBA; Brain; Mammary Cancer; Female Rat

\section{Introduction}

Female Sprague-Dawley (SD) rats develop mammary adenocarcinoma in response to a single intragastric dose of 7,12 dimethylbenz (a) Anthracene (DMBA), a polycyclic aromatic hydrocarbon (PHA) [1]. The mechanisms by which DMBA induces mammary tumours in rats have been well characterized. The carcinogen interacts with rapidly proliferating cells in the terminal end buds, forming DNA adducts, which in turn play a role in transforming normal terminal end bud cells into malignant cells [2-4]. The susceptibility of SD rats to DMBA peaks at 55-60 days of age and is eliminated by ovariectomy, suggesting that the inducible action of the carcinogen depends on ovarian hormones [5].

Furthermore, the development of DMBA-induced adenocarcinoma is preceded by a series of disturbances of the Hypothalamo-PituitaryGonadal (HPG) axis and the Hypothalamo-Pituitary-Adrenal axis (HPA) axes during the latency period. Along the HPG axis, a blunting of pre-ovulatory surges of plasma Luteinizing Hormone (LH) and Follicle-Stimulating Hormone (FSH) [6] together with an increase in the pre-ovulatory surge of plasma $17 \beta$-Estradiol $\left(\mathrm{E}_{2}\right)$ [7] were observed in response to DMBA. Also, female rats treated with DMBA displayed a reduction in GNRH1 release associated with a blunted $\mathrm{LH}$ release [8]. In addition, disturbances of the expression of the hypothalamic Gonadotropin-Releasing Hormone (GNRH1) and its pituitary receptor genes occur throughout the oestrus cycle [9]. In the HPA axis, 
a long-term deregulation of circadian and E2-induced corticosterone secretion was observed in DMBA-treated rats [10]. Also, DMBA induces a reduction of the secretion of melatonin from the Pineal Gland [11].

DMBA is a ligand for the Aryl Hydrocarbon Receptor (AHR) which is expressed in the central nervous system, including the Hypothalamus and the Hippocampus [12]. Upon DMBA binding, like in the presence of 2,3,7,8-Tetrachlorodibenzo-p-dioxin (TCDD), AHR translocates to the nucleus where it heterodimerizes with the AHR nuclear translocator (ARNT) and binds to AHR DNA recognition sites, also named xenobiotic response elements (XRE) [13]. Most AHR ligands display anti-estrogenic properties. This could be explained at the molecular levels by several mechanisms including estrogen catabolism, ubiquitination and degradation of both estrogen receptors, negative interaction between responsive elements or co-activator squelching [14]. However, recent studies showed that pro-estrogenic properties could also be observed according to the status of the estrogen receptor (bound versus unbound to its ligand) [15]. Interestingly, a co-localisation of AHR and ER $\beta$ was documented in the Anterior Pituitary [16] and the brain $[17,18]$.

Given the long-term dysregulation of the activity of HPG and HPA axes, two major neuroendocrine axes strongly positively and/ or negatively connected [19] and of the well documented effects of estrogens on the HPG and HPA axes, we hypothesized that DMBA can induce changes in specific area of the brain of the expression of genes encoding AHR, ERS1and ERS2 and induce the unbalanced functioning of the HPG and HPA axes and in the reduced melatonin secretion.

After DMBA administration, the expression of genes encoding AHR, ERS1 and ERS2 was registered in the Hypothalamus which is involved in the control of neuroendocrine functions, and in the Pineal Gland, which is involved in the control of Melatonin secretion.

The expression of genes encoding GNRH1, Corticotropin Releasing Hormone (CRH) and Hydroxy-Indo-O-Methyl-Transferase (ASMT), the rate limiting enzyme for the synthesis of Melatonin, were also recorded in the same extracts of Hypothalamus and Pineal Gland.

Last, the expression of all investigated genes was also recorded in the same extracts in the hippocampus, a structure of the brain which is not involved in the control of neuroendocrine functions, but in cognitive functions.

\section{Materials and Methods}

\section{Animals}

Ethics statement: The present study has been conducted according to the European rules and the CNRS instruction on Animal Welfare. The animal studies described in this manuscript were conducted in accordance with accepted standards of animal care. The study was approved by the ethical committee of Paris Descartes University. All surgery was performed under chloral anesthesia, and all efforts were made to minimize suffering.

SD female rats (Charles River) were purchased at 49 to 51 days of age. They were housed ( 5 animals per cage), under controlled temperature $\left(21+1^{\circ} \mathrm{C}\right)$. The animal housing room diurnal cycle was 12 hours light/12 hours' darkness (light on: from 7 a.m. to 7 p.m.) The animals receive UAR food and water at libidum.
After 6-8 days of acclimatation, the rats received, at 55-57 days of age, either $75 \mathrm{mg} / \mathrm{kg}$ of DMBA dissolved in $1 \mathrm{ml}$ of sesame oil or $1 \mathrm{ml}$ vehicle alone, on the day of estrous of the estrous cycle. Rats were treated at 55-57 days of life, since the induction of the mammary cancer is maximal at this time of life [1]. Then, DMBA-treated and control-treated rats were ovariectomized 5 days after administration of the treatments, to avoid a possible effect of variations of gonadal hormones on DMBA action. The ovariectomy was not performed before the administration of DMBA since the induction of the mammary tumors requires the presence of the ovary; when the ovary was present, the first palpable mammary tumors appear by 60 days after the induction of the carcinogenic process [6]. Rats were then sacrificed by decapitation one month later, at a time when there was still no palpable mammary tumor.

Then the Pineal Gland, the Hippocampus and the Hypothalamus (Hypothalami were removed using the anterior border of the optic chiasma, the posterior border of mammillary bodies and lateral hypothalamic sulci to a depth of $2.5 \mathrm{~mm}$ as dissection limits), were removed and immediately frozen in liquid nitrogen and stored at $-80^{\circ} \mathrm{C}$ until extraction.

Two series of experiments were performed, with 7 and 15 animals per DMBA-treated or Control-treated group, in the first and the second experiment, respectively.

\section{RNA extraction and RT-PCR}

Total RNA from the Pineal Gland, the Hippocampus and the Hypothalamus were obtained with the RNeasy mini kit for lipid tissues (Qiagen) according to the manufacturer's instructions. Sample quality was controlled with the $260 / 280 \mathrm{~nm}$ absorption ratio determination (Nano drop ND-1000, Thermo Scientific). For real-time PCR analysis, $1.25 \mu \mathrm{g}$ of total RNA was reverse transcribed with the multiscribe $\mathrm{T}^{\mathrm{TM}}$ reverse transcriptase of the high capacity cDNA archive kit (Applied Biosystems). cDNA were amplified on a thermal cycler (ABI-Prism 7900HT from Applied Biosystems) using specific oligonucleotides and the Sybr Green fluorescence method(SYBR Select Master MIX from Life Technologies). Specific sequences of forward and reverse primers were respectively as follows:

AHR: 5'-TTCCCTTATGAGTGCCTTG-3' and 5'CCTGAGTGTGTCTGATTTCC-3';

ERS1: 5'-TGCTCCTAACTTGCTCTTG-3' and 5'GATGTGGTCCTTCTCTTCC-3';

ERS2:5'-TGAGCACCTTGAGTCCAGAG-3' and CAGTCCCACCATTAGCACC-3';

CRH: 5'-CTGTCCCCCAACTCCAC-3' and 5'GCTAACTGCTCTGCCCTG-3';

GNRH1: 5'-CTGGTCCTATGGGTTGC-3' 5 and
AGTTCTGGGGTCTGCC-3';
ASMT: 5 '-CTCCTGCTGTCACTCAAC-3' and 5'-
CAAAACTTCCTGTCCCC-3';

For each couple of primers, PCR efficiency and specificity were tested with serial dilutions of samples and melting curve analysis, respectively. Data were normalized with the ribosomal 18S RNA level as a reference and quantification was carried out by comparison of the number of cycles required to reach reference and target threshold values ( $\delta$ - $\delta$ Ct method). 
Citation: $\quad$ Cadoudal T, Lenoir V, Penot G, Sathish ISH, Zhao Y, et al. (2014) Decrease in Aryl Hydrocarbon Receptor and 17ß-Estradiol Receptor (A\&B) Gene Expression in The Hypothalamus and The Pineal Gland, After Administration of Dimethylbenz (A) Anthracene, A Mammary Carcinogen, To Sprague-Dawley Female Rats.. J Steroids Hormon Sci 5: 127. doi:10.4172/2157-7536.1000127

Page 3 of 5

\section{Statistical analysis}

The main purpose of this study was to compare the gene expression between control group and DMBA group within the Pineal Gland, the Hippocampus and the Hypothalamus. Results of two independent experiments were pooled before statistical analysis. Mean and standard deviation were presented with $95 \%$ confidence interval. The MannWhitney test was performed to compare gene expression between control and DMBA group. All analyses were performed in SAS 9.1 (Cary, NC). The significance level was at least 0.05 .

\section{Results}

Results are shown in figures 1 to 3.

In the PINEAL GLAND (Figure 1), mRNA levels of the AHR, ERS1, ERS2, GNRH1 and ASMT were significantly different between DMBA and control groups. Transcript abundance in the DMBA group was lower than in controls for AHR, ERS1 and ERS1. However, transcripts from DMBA-treated animals were more abundant than in the control group for GNRH1 and ASMT. All other mRNA levels were not found significantly different between DMBA and control groups.

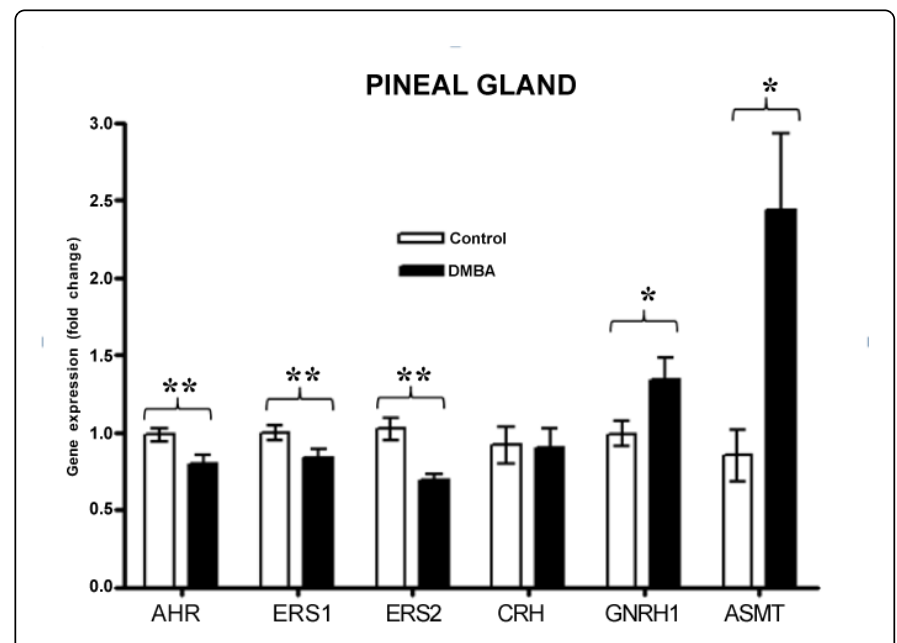

Figure 1: Relative gene expression levels within the Pineal Gland.

Expression levels were normalized to those of 18S. Empty bars indicate the normalized levels of gene expression for the control group. Asterisks represent statistically-significant differences between the DMBA group and the corresponding control group.

Mean \pm SE of 18-20 determinations

${ }^{*} p<0.05 ;{ }^{* *} p<0.01$

In the HIPPOCAMPUS (Figure 2), the expression of all investigated genes was similar in DMBA and control groups.

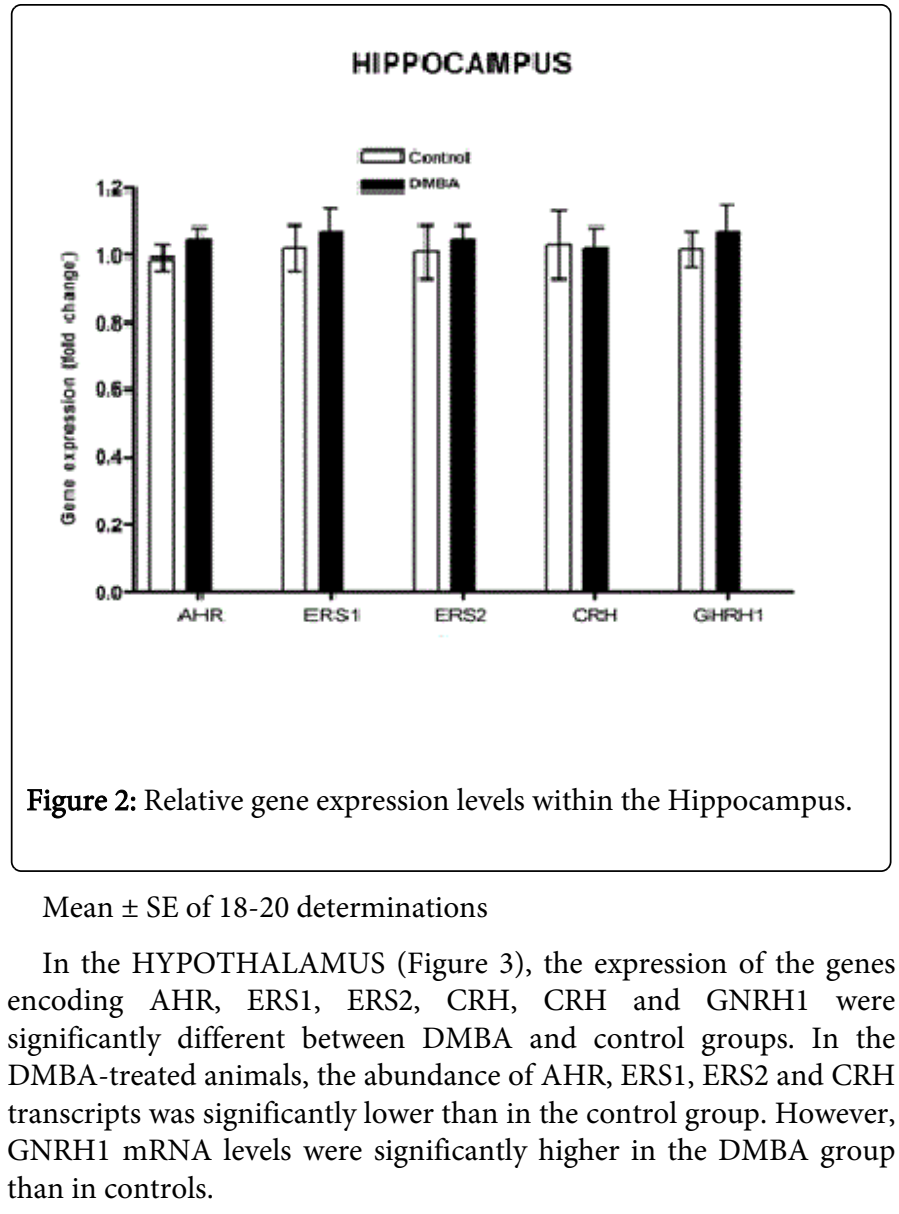

than in controls.

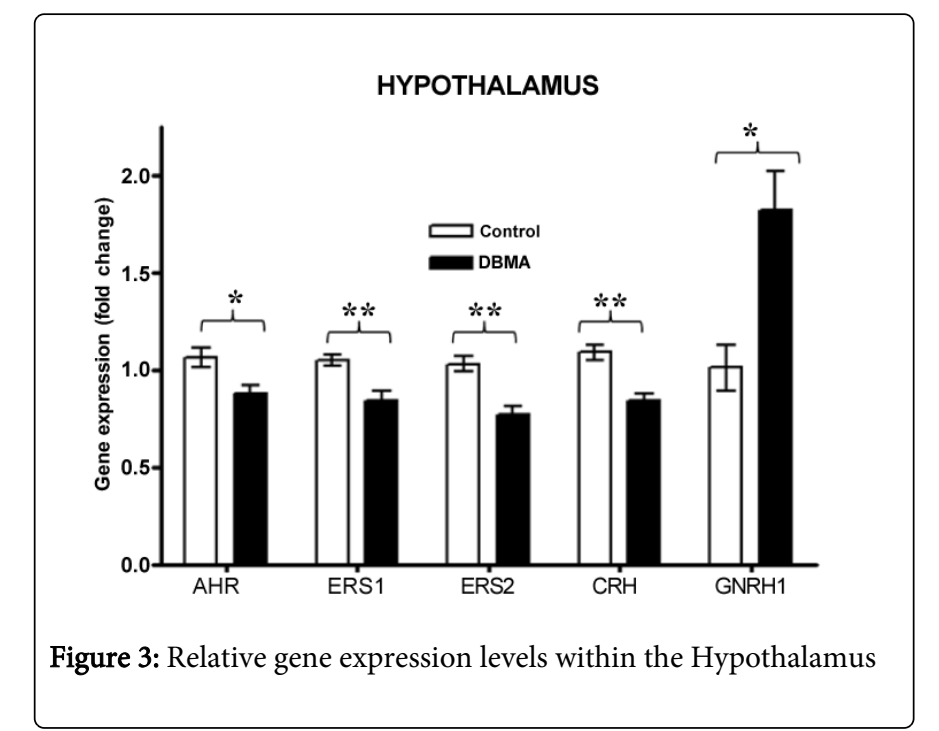

Expression levels were normalized to those of 18S. Empty bars indicate the normalized levels of gene expression for the control group. Asterisks represent statistically-significant differences between the DMBA group and the corresponding control group.

Mean \pm SE of 18-20 determinations

${ }^{*} P<0.05 ;{ }^{* *} p<0.01$ 


\section{Discussion}

The present results clearly provide strong evidence that changes in brain gene expression were observed only in the Pineal Gland and the Hypothalamus one month after exposure to DMBA. An almost identical parallel decrease was recorded for the expression of AHR, ERS1 and ERS2 genes. The long-term decrease in the expression of the AHR gene and in the expression of both ERS1 and ERS2 genes is probably due to the interaction of DMBA with these three receptors. Indeed, DMBA is an AHR ligand that rapidly leads to nuclear translocation of the receptor and to transcriptional activation of target genes after its heterodimerization with ARNT. However, this process also causes the degradation of the AHR, which is sometimes described as a negative feedback. This mechanism might not be sufficient to explain the observed down-regulation because DMBA is not a persistent organic pollutant and this negative feedback might be then difficult to maintain after one month which is the period between DMBA treatment and the sacrifice of animals.

Most AHR ligands were also historically described as anti-estrogens [14]. For example, female Sprague-Dawley rats treated with TCDD, an AHR high affinity ligand, display delayed incidence of mammary tumors [20]. Only few studies were carried out on the nervous system of mammals with DMBA [21] but alternative ligands, including TCDD, were sometimes used. Experimental studies showed that TCDD-exposed animals display multiple symptoms including defects of motor coordination or spatial memory [22-24]. The associated mechanisms have not been discovered yet but the anti-estrogenic properties of AHR ligands might be important to consider; estrogens and ERs indeed regulate several developmental or homeostatic process related to the nervous system [25]. The interactions between the AHR and ERs have been characterized at the molecular level and might be considered to understand the effects of DMBA on ERs in our study. First, AHR ligands increase the expression of several cytochromes P450 including CYP1A1 and CYP1B1 which catabolize $17 \beta$-Estradiol and decrease its concentration [12,26,27]. Second, both AHR and ERs share common co-activators (CBP/p300); competition might occur if both signaling pathways are simultaneously activated [28]. Third, the dioxin receptor responsive element (XRE) was early on described in the promoter of $17 \beta$-Estradiol responsive genes [28]. The proximity of the XRE with the $17 \beta$-Estradiol response element very likely supports interactive action between AHR, ERS1 and ERS2 [16].

Finally, recent studies highlighted a new property of AHR, showing that the receptor also forms a nuclear complex with ERS1 and CUL4B (an E3 ubiquitin ligase) triggering the ubiquitination of ERS1 and subsequently its degradation [29]. Interestingly, this is only observed if ERS1 is bound to $17 \beta$ - Estradiol; conversely, if ERS1 is unbound, AHR acts as a co-activator on estrogen-responsive promoters suggesting that the interaction between both receptors is more complex than previously described; this AHR might be alternatively described as a modulator of estrogenic pathways [15]. A co-localization of the AHR with ERS2 was already demonstrated at the brain level, thus suggesting that an interaction might occur between these receptors [30,31]. The decreased expression of ERS1 and ERS2 in our study might be due to an activated AHR pathway that stimulates the ubiquitination process. Alternatively, genetic or epigenetic mechanisms could also take place to explain the long-term effect of DMBA and needs to be explored because it could represent a novel type of crosstalks between AHR and ERs.
DMBA was previously shown to mimic most of the biological effects of $17 \beta$-Estradiol [32] and its effects on ERs were documented [33].

The DMBA effects on GNRH1, ASMT and CRH transcripts most likely result from the direct or indirect action of DMBA throughout changes in AHR and ERs gene expression.

At the hypothalamic level, the reverse relationship between the expression of GNRH1 and CRH genes is in agreement with the welldocumented reverse relationship between the activity of HPG and HPA axes [19]. Interestingly, the expression of the ASMT gene, encoding the rate-limiting enzyme for the synthesis of melatonin, was markedly up-regulated by DMBA. However, DMBA reduces the secretion of melatonin from the Pineal Gland [11].

All together the most important findings of the present study are that DMBA induces identical long term decreases in the expression of AHR, ERS1 and ERS2 genes, in the Pineal Gland and the Hypothalamus, two neuroendocrine structures, whereas no effect is detected at the Hippocampus level, a non-neuroendocrine structure.

Whether or not the decreases seen for the expression of AHR, ERS1 and ERS2 genes in the Pineal Gland and the Hypothalamus play a role in the promotion of the carcinogenic process remains to be demonstrated.Follow up studies will involve protein expression of the investigated genes.

\section{Acknowledgment}

We thank Krystel El Hage for her help during the preparation of the manuscript.

\section{References}

1. Huggins C, Grand LC, Brillantes FP (1961) Mammary cancer induced by a single feeding of polymucular hydrocarbons, and its suppression. Nature 189: 204-207.

2. Daniel FB, Joyce NJ (1983) DNA adduct formation by 7,12dimethylbenz[a]anthracene and its noncarcinogenic 2-fluoro analogue in female Sprague-Dawley rats. J Natl Cancer Inst 70: 111-118.

3. Russo J, Tait L, Russo IH (1983) Susceptibility of the mammary gland to carcinogenesis. III. The cell of origin of rat mammary carcinoma. Am J Pathol 113: 50-66.

4. Russo J, Tay LK, Russo IH (1982) Differentiation of the mammary gland and susceptibility to carcinogenesis. Breast Cancer Res Treat 2: 5-73.

5. Dao TL (1962) The role of ovarian hormones in initiating the induction of mammary cancer in rats by polynuclear hydrocarbons. Cancer Res 22: 973-981.

6. Kerdelhue B, El Abed A (1979) Inhibition of preovulatory gonadotropin secretion and stimulation of prolactin secretion by 7,12dimethylbenz(a)anthracene in Sprague-Dawley rats. Cancer Res 39: 4700-4705.

7. el Abed A, Kerdelhue B, Castanier M, Scholler R (1987) Stimulation of estradiol-17 beta secretion by 7,12-dimethylbenz (a) anthracene during mammary tumor induction in Sprague-Dawley rats. J Steroid Biochem 26: 733-738.

8. Kerdelhue B, Peck EJ Jr (1981) In vitro LHRH release: correlation with the LH surge and alteration by a mammary carcinogen. Peptides 2: 219-222.

9. Jakubowski $M$, Lenoir $V$, Jimenez-Linan $M$, Duval $P$, Israel $L$, et al. (2002) Long-term effects of the mammary carcinogen 7,12dimethylbenz(a) anthracene on hypothalamic gonadotropin-releasing hormone and its pituitary receptor gene expression, during the 
Citation: Cadoudal T, Lenoir V, Penot G, Sathish ISH, Zhao Y, et al. (2014) Decrease in Aryl Hydrocarbon Receptor and 17ß-Estradiol Receptor (A\&B) Gene Expression in The Hypothalamus and The Pineal Gland, After Administration of Dimethylbenz (A) Anthracene, A Mammary Carcinogen, To Sprague-Dawley Female Rats.. J Steroids Hormon Sci 5: 127. doi:10.4172/2157-7536.1000127

Page 5 of 5

promotion stage, in female Sprague-Dawley rats. Breast Cancer Res Treat 73: 23-29.

10. Yon de Jonage-Canonico MB, Lenoir V, Scholler R, Kerdelhué B (2005) Long-term dysregulation of circadian and 17-beta estradiol-induced LH, prolactin and corticosterone secretion after dimethylbenz (a) anthracene administration in the Sprague-Dawley female rat. Breast Cancer Res Treat 92: 47-50.

11. De Jonage-Canonico MB, Lenoir V, Martin A, Scholler R, Kerdelhué B (2003) Long term inhibition by estradiol or progesterone of melatonin secretion after administration of a mammary carcinogen, the dimethyl benz(a)anthracene, in Sprague-Dawley female rat; inhibitory effect of Melatonin on mammary carcinogenesis. Breast Cancer Res Treat 79: 365-377.

12. Huang P, Rannug A, Ahlbom E, Håkansson H, Ceccatelli S (2000) Effect of 2,3,7,8-tetrachlorodibenzo-p-dioxin on the expression of cytochrome $\mathrm{P} 450$ 1A1, the aryl hydrocarbon receptor, and the aryl hydrocarbon receptor nuclear translocator in rat brain and pituitary. Toxicol Appl Pharmacol 169: 159-167.

13. Barouki R, Aggerbeck M, Aggerbeck L, Coumoul X (2012) The aryl hydrocarbon receptor system. Drug Metabol Drug Interact 27: 3-8.

14. Coumoul X (2007) [Dioxin and estradiol, a "complex" story]. Med Sci (Paris) 23: 701-702.

15. Ohtake F, Takeyama K, Matsumoto T, Kitagawa H, Yamamoto Y, et al (2003) Modulation of oestrogen receptor signalling by association with the activated dioxin receptor. Nature 423: 545-550

16. Ruegg J, Swedenborg E, Wahlstrom D, Escande A, Balaguer P, et al. (2008) The transcription factor aryl hydrocarbon receptor nuclear translocator functions as an estrogen receptor beta-selective coactivator, and its recruitment to alternative pathways mediates antiestrogenic effects of dioxin. MolEndocrinol 22: 304-316.

17. Frye CA, Bo E, Calamandrei G, Calzà L, Dessì-Fulgheri F, et al. (2012) Endocrine disrupters: a review of some sources, effects, and mechanisms of actions on behaviour and neuroendocrine systems. J Neuroendocrinol 24: $144-159$

18. Kajta M, WÃ $\tilde{3}^{3}$ towicz AK, MaÄłkowiak M, LasoÅ, W (2009) Aryl hydrocarbon receptor-mediated apoptosis of neuronal cells: a possible interaction with estrogen receptor signaling. Neuroscience 158: 811-822.

19. Kerdelhue B, Jones GS, Gordon K, Seltman H, Lenoir V, et al. (1995) Activation of the hypothalamo-anterior pituitary corticotropin-releasing hormone, adrenocorticotropin hormone and beta-endorphin systems during the estradiol 17 beta-induced plasma LH surge in the ovariectomized monkey. J Neurosci Res 42: 228-235.

20. Holcomb M, Safe S (1994) Inhibition of 7,12-dimethylbenzanthraceneinduced rat mammary tumor growth by 2,3,7,8-tetrachlorodibenzo-pdioxin. Cancer Lett 82: 43-47.
21. Granberg L, Ostergren A, Brandt I, Brittebo EB (2003) CYP1A1 and CYP1B1 in blood-brain interfaces: CYP1A1-dependent bioactivation of 7,12-dimethylbenz(a)anthracene in endothelial cells. Drug MetabDispos 31: $259-265$

22. Nishijo M, Kuriwaki J, Hori E, Tawara K, Nakagawa H, et al. (2007) Effects of maternal exposure to 2,3,7,8-tetrachlorodibenzo-p-dioxin on fetal brain growth and motor and behavioral development in offspring rats. ToxicolLett 173: 41-47.

23. Seo BW, Sparks AJ, Medora K, Amin S, Schantz SL (1999) Learning and memory in rats gestationally and lactationally exposed to $2,3,7,8$ tetrachlorodibenzo-p-dioxin (TCDD). NeurotoxicolTeratol 21: 231-239.

24. Thiel R, Koch E, Ulbrich B, Chahoud I (1994) Peri- and postnatal exposure to 2,3,7,8-tetrachlorodibenzo-p-dioxin: effects on physiological development, reflexes, locomotor activity and learning behaviour in Wistar rats. Arch Toxicol 69: 79-86.

25. Carpenter KD, Korach KS (2006) Potential biological functions emerging from the different estrogen receptors. Ann N Y Acad Sci 1092: 361-373.

26. Coumoul X, Diry M, Robillot C, Barouki R (2001) Differential regulation of cytochrome P450 1A1 and 1B1 by a combination of dioxin and pesticides in the breast tumor cell line MCF-7. Cancer Res 61: 3942-3948.

27. Tsuchiya Y, Nakajima M, Yokoi T (2005) Cytochrome P450-mediated metabolism of estrogens and its regulation in human. Cancer Lett 227: $115-124$.

28. Safe S, Wormke M, Samudio I (2000) Mechanisms of inhibitory aryl hydrocarbon receptor-estrogen receptor crosstalk in human breast cancer cells. J Mammary Gland Biol Neoplasia 5: 295-306.

29. Ohtake F, Baba A, Takada I, Okada M, Iwasaki K, et al. (2007) Dioxin receptor is a ligand-dependent E3 ubiquitin ligase. Nature 446: 562-566.

30. Baba T, Mimura J, Nakamura N, Harada N, Yamamoto M, et al. (2005) Intrinsic function of the aryl hydrocarbon (dioxin) receptor as a key factor in female reproduction. Mol Cell Biol 25: 10040-10051.

31. Cao J, Patisaul HB, Petersen SL (2011) Aryl hydrocarbon receptor activation in lactotropes and gonadotropes interferes with estradioldependent and -independent preprolactin, glycoprotein alpha and luteinizing hormone beta gene expression. Mol Cell Endocrinol 333: 151-159.

32. Pasqualini C, Sarrieau A, Dussaillant M, Corbani M, Bojda-Diolez F, et al. (1990) Estrogen-like effects of 7,12-dimethylbenz(a)anthracene on the female rat hypothalamo-pituitary axis. J Steroid Biochem 36: 485-491.

33. Garcia-Segura LM, Diolez-Bojda F, Lenoir V, Naftolin F, Kerdelhué B (1992) Estrogen-like effects of the mammary carcinogen 7,12dimethylbenz(alpha)anthracene on hypothalamic neuronal membranes. Brain Res Bull 28: 625-628. 